\begin{tabular}{|l|l|}
\hline$E A-7$ & 211 \\
\hline
\end{tabular}

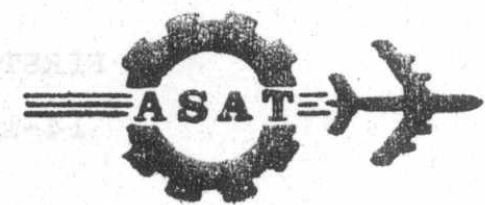

MILITARY TECHNICAL COLLEGE

CAIRO - EGYPT

\title{
MEASUREMENTS OF MEAN FLOW VELOCITY PROFILES \\ OVER ROTATCNG SMOOTH AND ROUGH DISKS
}

\author{
M.A. ElRefaie, M.M. Kemry, ${ }^{* *}$ M.A. Halawa ${ }^{\star \star *}$
}

\section{ABSTRACT}

Measurements of the mean flow velocities over a disk rotating in a quiescent air are presented in this work. The measurements were performed on flat and rough disk surfaces such that the effect of surface roughness can be investigated. The surface roughnessemployed in the present work was $0.03 \mathrm{~mm}$. Measurements were extended from the laminar region to the turbulent region where the maximum value of Reynolds number employed in the present investigation is $3.7 \cdot 10^{5}$. A hot wire anemometer with a single sensing wire was used in the measurements. The three components of the mean velocity were separated by performing measurements in two distinct directions of the sensing wire. The transition region was detected by employing an oscilloscope jouned to the anemometer circuit.

The results indicate that the boundary layer velocity is composed mainly of radial and tangential components in the laminar region. In the transition and turbulent regions, away from the disk surface by a small distance, the magnitudes of the three components of velocity are of the same order. The effect of surface roughness is to cause the transition region to start at smaller values of Reynolds number and to increase the magnitudes of the three components of velocity in the transition and turbulent regions. Comparisons between this work and previous works are presented and discussed.

* Professor, ** Assistant professor, *** Demonstrator, Mech. Eng. Dept, Al-Azhar University, Cairo, Egypt. 


\section{INTRODUCTION}

The flow over a finite disk rotating in its own plane in an otherwise quiescent air is a complicated three dimenensional one. Near the disk axis is a region of laminar boundary layer flow. At large radii, there is a region of transition flow. At even greater radii, a fully developed turbulent boundary layer occurs.

The laminar flow over an infinite disk was investigated by Von Kármán [1] and the solution was improved by Cochran [2]. Cham 8. Head [3] and Cooper [4] solved the turbulent boundary layer equations with empirical correlations based on two dimensional flow. Their solutions were greatly improved over the solutions of Von Kármán [1] and Goldstien [5] whose correlations were not based on experiments. However, these improved solutions were not compatible with the experiments [3]. This was due to the ignoring of the axial velocity component in the theor etical works. The previous measurements of the laminar and turbulent boundary layers on a rotating disk were not comprehensive. Earlier experiments were performed by Theodorsen and Regier [6] who measured the torque produced from the turbulent boundary layer. Kreith and Taylor [7] measured the mass transfer rates and the friction coefficients in the laminar and turbulent regions. Cham and Head [3] measured the frictional torque and the velocity components in the radial and tangential directions of turbulent flow. In their measurements, they ignored the axial velocity component.

The cbject of the present investigation is to obtain reasonably complete and detailed measurements of the mean components of velocity in the three regions of flow. This includes the measurements of the axial velocity component which has been ignored by previous workers. In addition, the effect of the roughness of the disk surface on the flow is also investigated.

\section{VELOCITY VECTOR ANALYSIS AND MEASURING TECHNIQUE}

Boundary layer measurements were made by using a hot wire anemometer with a single sensing wire. Separation of the three components of velocity require the measurements to be performed in distinct positions of the sensing wire. This is discussed in the present section.

The paper of Jorgnesen [8] showed that two factors $k_{1}$ and $k_{2}$ are important when a single hot wire probe is placed in a three dimensional velocity field and reference to fig. 1 should serve to clarify this point. Two planes of particular concern are illustrated in the figure. Plane (1) is defined by the sensing wire and the probe axis. Plane (2) is perpendicular to plane (1) and passes through the probe axis. The probe is calibrated in the position where $\phi=0$. Considering $V$ flowing along the probe axis, this velocity will be registered as being of the same magnitude of $V_{\text {eff }}$. Now if $\phi$ is not equal to zero and $V$ is allowed to rotate about the probe axis as shown in fig.1, the cooling effect is greatly influenced by the rotation of $q$ while $w$ has no influence since it lies in the same direction of calibration. In general $V$ is not equal to $V$. If $V$ lies in plane (1) $(\alpha=0)$, the cooling effect of $q$ will be minimum because $q$ is tangent to the sensing wire. For this reason a yaw factor $k_{1}$ has to be included as a correction factor for the tangential 
cooling velocity. If $V$ lies in plane (2) $\left(\alpha=90^{\circ}\right)$, the cooling effect of $q$ will be maximum, since $q$ is normal to the wire, and for this reason a second factor $K_{2}$, the pitch factor, is required to correct for the velocity in plane (2). Referring to fig. 1 and using the two factors described above, the effective colling velocity $V_{\text {eff }}$ acting on the sensor at any angle a can be expressed as

$v_{\text {eff }}^{2}=w^{2}+k_{1}^{2} q_{1}^{2}+k_{2}^{2} q_{2}^{2}$

Where $q_{1}$ and $q_{2}$ are the components of $q$ in planes (1) and (2) respectively. Eq. (1), known as the characteristic equation, can be written in the form

$v_{\text {eff }}^{2}=w^{2}+q^{2}\left(k_{1}^{2} \cos ^{2} \alpha+k_{2}^{2} \sin ^{2} \alpha\right)$

In order to apply Eq. (2) at any angle $\alpha$, the values of $V_{\text {eff }}, K_{1}$, and $K_{2}$ must be known. This was attained by performing the required correlations in a wind tunnel where $V$ was measured by a standard pitot tube. The probe equation is given by

$e^{2}-e_{0}^{2}=B V_{e f f}^{n}$

Calibration between $v_{e f f}$ and $\left(e^{2}-e_{0}^{2}\right)$ was performed by aligning the probe axis in a direction parallel to $V$. In that position, $V_{\text {eff }}=V$. The results are shown in Fig. 2 and the values of $B$ and $n$ are 6.11 and 0.46 respectively. The probe axis was then turned such that it became normal to $V$ and its sensor was tangent to $V$. In this position $V_{\text {eff }}=K_{1} V$. The correlation between $K_{1}$ and $V$ was achieved by knowing the values of $e_{0}$, $e$, $\mathrm{V}, \mathrm{B}, \mathrm{n}$, and employing Eq. (3). To obtain correlation between $\mathrm{K}_{2}$ and $\mathrm{V}$, the sensor was turned by $90^{\circ}$ about its axis from the above position $\left(V_{\text {eff }}=K_{2} V\right)$ and similar procedure was performed. The results are shown in Fig. 3 .

To enable the three components of velocity to be separated, measurements were carried out in the two positions shown in Fig.1 (notice that in the measurements, the sensor was rotated about its axis until it lies in plane (1) or plane (2), while $V$ takes a fixed direction). Application of Eq.(2) to the first position where $\alpha=0$, yields

$v_{\text {eff }}^{2}(1)=w^{2}+k_{1}^{2} q^{2}$

Application of Eq. (2) to the second position where $\alpha=90^{\circ}$, yields

$v_{\text {eff }}^{2}(2)=w^{2}+k_{2}^{2} q^{2}$

The above two positions were identified by the readings of the registered voltages, where they are of minimum and maximum values. $V_{\text {eff }}(1)$ and $V_{\text {eff }}(2)$ can be obtained from Fig.2. Equations (4) and (5) can be solved for $w$ and $q$. knowing the yaw angle at any one of the above positions, $u$ and $v$ can be computed. It is to be noticed that the solution to equations (4) and (5) is obtained by iteration, since both $k_{1}$ and $k_{2}$ are functions of $V$ as shown in Fig. 3 . 


\section{APPARATUS}

The main parts of the apparatus are simply a wooden disk $(R=24.35 \mathrm{~cm}$, $t=1.3 \mathrm{~cm}$ ) and a sensing wire placed at a location where the velocity is measured. The disk is rotating in a horisontal plane and the wire is parallel to it. The probe axis is normal to the disk surface. The disk is connected to a vertical shaft which is aligned by two ball-bearings, $30 \mathrm{~cm}$ apart. The shaft is driven by a $2 \mathrm{hp}$ motor via pullies and a V-belt. The rotational speed can be varied by employing pullies of variable diameters, and it is measured by a stroboscope. The maximum disk rotational speed attained in the present work is $880 \mathrm{rpm}$. The probe axis, which carry the sensing wire, is mounted on the lower end of a traversing mechanism. The later can move in both radial and vertical directions. The radial motion of the traversing mechanism is recorded by a metering scale of $0.5 \mathrm{~mm}$ accuracy, and its axial movement is recorded by a dial gage of $0.01 \mathrm{~mm}$ accuracy. The yaw angle is measured by a protractor of $0.5^{0}$ accuracy, placed parallel to the disk surface and can rotate with the probe axis. Precautions were made such that vibration was avoided to a great extent. In addition to the above mentioned parts of the apparatus, an oscilloscope joined to the anemometer circuit was used to detect the transition region of flow.

\section{RESULTS AND DISCUSSIONS}

Experiments were performed on smooth and rough disk surfaces. For smooth disk, the surface was flat to $0.15 \mathrm{~mm}$. Rough surface was obtained by placing sand papers of mean roughness $0.03 \mathrm{~mm}$ on the smooth disk surface. The transition regions were detected at various speeds. The results indicated that there are approximately constant values of $R_{e}$ at which transitions from laminar to transition regions and from transition tp turbulent regions occur. For smooth surface, these values are $1.8 * 10^{5}$ and $2.8 * 10^{5}$ respectively, and for rough surface, they are $1.4 * 10^{5}$ and $2.2 * 10^{5}$ reserectively (see Fig.9).

Fig.4 shows the velocity profiles in the laminar region for two different values of $R_{e}$. It is seen from this figure that the profiles are identical and hence a single profile at any value of $\mathrm{R}_{\mathrm{e}}$ can represent the solution in the whole region. This supports the laminar $e$ thecry of Von Kármán [1]. The theoritical solution of Cochran [2] is also presented in the figure. The agreement between theory and experiment is good everywhere which indicates that the calibration process and the experimental accuracy of the present work is satisfactory. As seen from the figure, the magnitude of $w$ is very small as compared with the other two components. The surface roughness has no effect on the profiles up to a value of $R_{e}=1.4 * 10^{5}$ where transition occurs over rough disk surface.

Figures 5,6, and 7 illustrate the profiles of $\bar{w}, \bar{v}$, and $\bar{u}$ in the transition and turbulent regions respectively. As seen, the effect of surface roughness is to increase the magnitudes of all velocity components. The profiles in the transition and turbulent regions are of the same behaviour. Fig.4 shows that $\bar{w}$ vanishes over the disk surface by an axial distance which increases by increasing $R_{e} \cdot \bar{w}$ is increased by increasing $\bar{z}$ until it reaches a peak value $\bar{w}_{p}$ at a location $\bar{z}_{p}$. The magnitudes of $\bar{w}_{p}$ and $\bar{z}_{p}$ are shown to increase by increasing $R_{e}$. Fig. 7 shows that, near the disk surface, $\bar{u}$ increases with high rates $\epsilon^{\circ}$ until it reaches a peak value $\bar{u}_{p}$ at a small distance 
of $\bar{z} \cdot \bar{u}_{\mathrm{p}}$ is shown to decrease by increasing $R_{\text {until }}$ it reạches a constant value of about 0.12 in the turbulent region (Notice that although $\bar{u}_{p}$ is decreased by increasing $R_{e}$, its dimensional value increases). Fig. 6 shows that near the disk surface, the profiles of $\bar{v}$ become more flat as $R_{e}$ increases. Figures 5 to 7 show that the boundary layer thickness $\bar{\delta}$ increases by increasing $R$. At the edge of the boundary layer, the magnitudes of $\bar{u}$ and $\bar{v}$ approach zero, while that of $\bar{w}$ has small magnitude which does not vanish since there must be an axial inflow to feed the radial outflow at the disk periphery.

A comparison between the velocity profiles of the present work and of previous workers is shown in Fig.8 (for smooth surface). Figure 8-a shows that the agreement between experiments is good. However, there is a disagreement between theory and experiments in the range $3<\bar{z}<16$ (notice the disagreement between theory and experiment of the same reference). The agreement shown in Fig. 8-b is good everywhere except at the region of the outer layer. The disagreement between theory and experiment is due to the fact that the axial momentum equation was ignored in the thearetical work. This assumption is only correct at small values of $\bar{z}$ where $\bar{w}$ vanishes or has small magnitudes. At intermediate and high values of $\bar{z}$, the magnitude of $\bar{w}$ is of the same order of $\bar{u}$ and $\bar{v}$, and thus the above mentioned assumption can not be applied. Boundary layer parameters are shown in Figures 9 and 10 . The theoritical solutions (for flow over smooth disks) of Von Kármán [1] and Cooper [4] are also presented. The agreement of all works is only seen at large values of $R_{e}$. Von Kármán assumed that the flow is fully turbulent allover the disk surface. This indicates the reason of the big departure between his results and the present results at low values of $R_{e}$ and as seen the departure is decreased by increasing $R_{e}$. This means that the solution of Von Kármán is only correct at high values of $R_{e}$. The same conclusion were obtained by Cham and Head [3]. The solution of cooper was based on the assumption that the flow is laminar up to a value of $\mathrm{R}_{e}=3.04 * 10^{5}$ and above this value the flow is fully turbulent. The agreement between the present work and that of Cooper is seen in the laminar and turbulent regions, and the disagreement is shown in the transition region which was neglected by cooper, who mentioned that his results were not correct in this region. As seen from the figures, the effect of surface roughness is to increase $\overline{\sigma^{*}}$ and $\mathrm{H}$ in the turbulent region.

\section{CONCLUSIONS}

In the present study, it was shown that a hot wire anemometer with a single sensing wire can be used to measure the mean velocity components in the three cylindrical directions. Analysis of the directional characteristics of the anemometer showed that the three components can be separated by performing measurements in two distinct positions of the sensing wire.

From the measurements performed in the present study, the following important results are concluded: For a disk with a smooth surface, the laminar region is extended from the disk axis to a radius corresponding to a value of Reynolds number of $1.8^{*} 10^{5}$. In this region, the velocity is composed mainly of radial and tangential components; the profiles are identical at any value of $\mathrm{Re}$; and at any speed, the boundary layer thickness is constant allover the region. 
In the transition and turbulent regions, the velocity profiles are of the same behaviour; the boundary layer thickness is increased by increasing $R_{e}$; and the axial velocity component vanishes over a small axial distance from the disk surface. However, at further axial distance, the three components of velocity have the same order of magnitudes. The surface roughness has no effect on the flow in the laminar region, but this region ends at earlier radial positions when reugh disk surface is used. The effect of surface roughness on the other two regions of flow is to increase the magnitudes of all components of velocity.

\section{REFERENCES}

1. Von Kármáiı Th., "Uber laminar und turbulent Reibung", Zeitschrift Fur angewanute Mathematic und Mechanik, Vol.1, No.4. (1921)

2. Cochran, W.G., "The flow due to a Rotating Disk", Proc.camb. Phil.Soc. $30,365-375$ (1934)

3. Cham, T.S. and Head, M.R., "Turbulent Boundary-Layer Flow on a Rotating Disk", J. of fluid Mech., Vol. 37, Pt. 1,129-147 (1969)

4. Cooper, P. "Turbulent Boundary-Layer on a Rotating Disk Calculated with an Effective Viscosity", AIAA J. Vol.9, No.2, 255-261 (1971)

5. Goldstein, S., "On the Resistance to the Rotation of a Disk Immersed in a Fluid", Proc. Camb. Phil. Soc. 31. (1935)

6. Theodorsen, T. and Regier, A. "Experiments on Drags of Revolving Disks, Cylinders, and Streaml ine Rods at High Speeds," NACA, Rept. No.793 (1944)

7. Kreith, F. and Taylor, J.H., "Heat and Mass Transfer From a Rotating Disk", J. of Heat Transfer (1959)

8. Jorgensen, F.E., "Directional Sensitivity of Wire and Fiberfilm Probes", DISA Information, No. 11,31-37 (1971)

\section{NOMENCLATURES}

e output voltage, volts

$\epsilon_{c} \quad$ Voltage at stagnant conditions

H Shape factor

$k_{1}$ yaw factor

$\mathrm{K}_{2} \quad$ Pitch factor

Re Reynolds number $=\omega r^{2} / v$

$r$ radius from axis of rotation

$V$ magnitude of absolute velocity $\mathrm{m} / \mathrm{s}$

Veff: effective cooling velocity, $\mathrm{m} / \mathrm{s}$

u,v,w mean velocity camponents in radial, tangential, and axial directions, respectively

$\bar{u}, \bar{v}, \bar{w}$ the values of $u, v$, and $w$ divided by $\omega r$

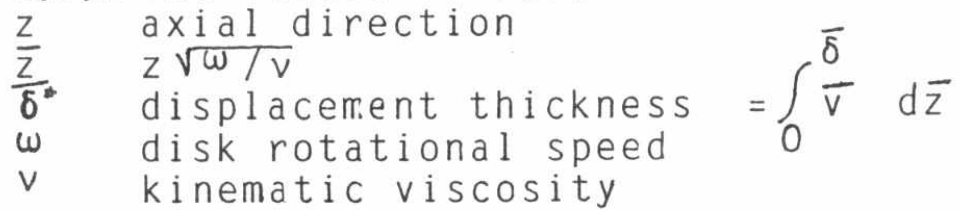




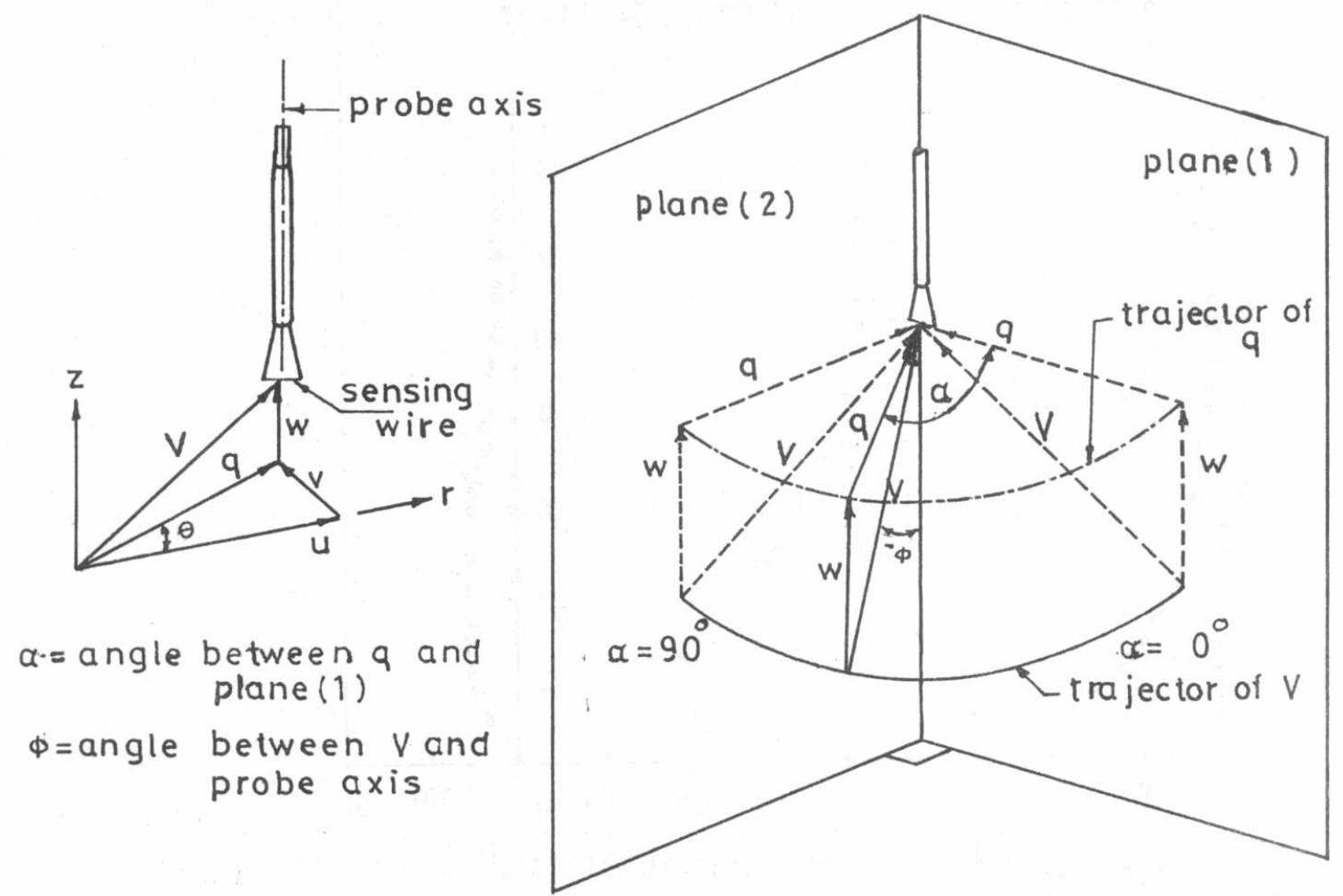

Fig.1. Directional characteristics of the hot wire anemometer

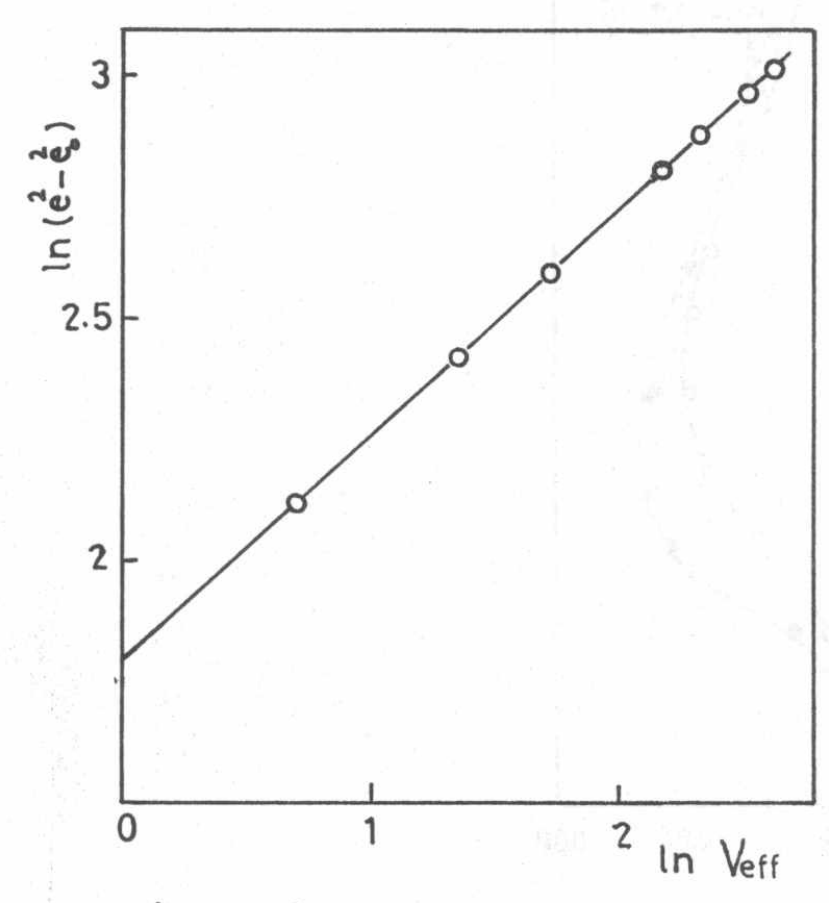

Fig.2. Calibration of hot wire

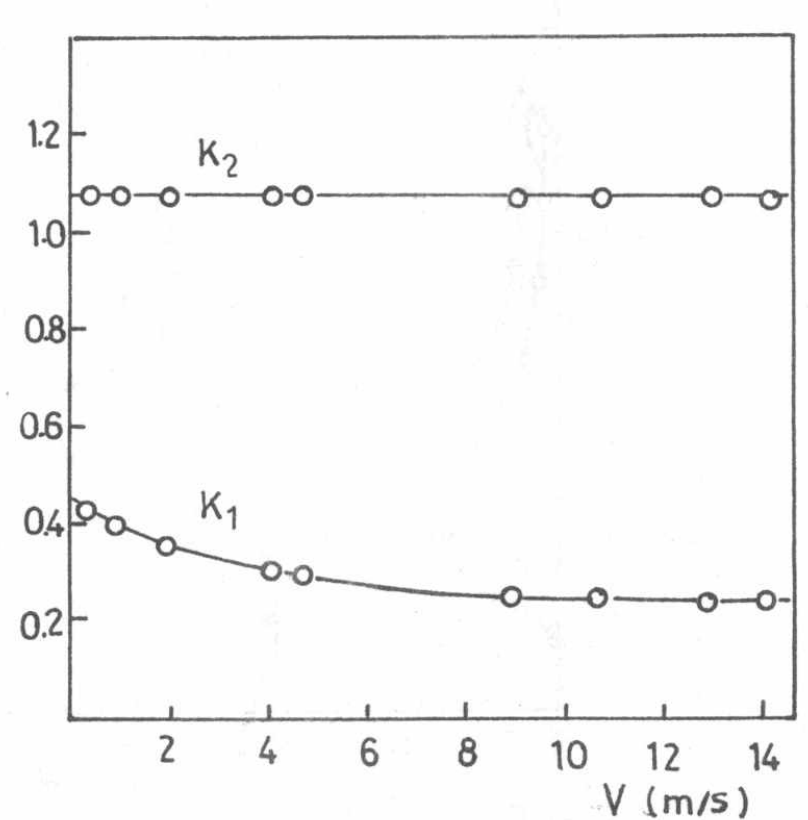

Fig.3. Correction factors, $k_{1}$ and $k_{2}$ 


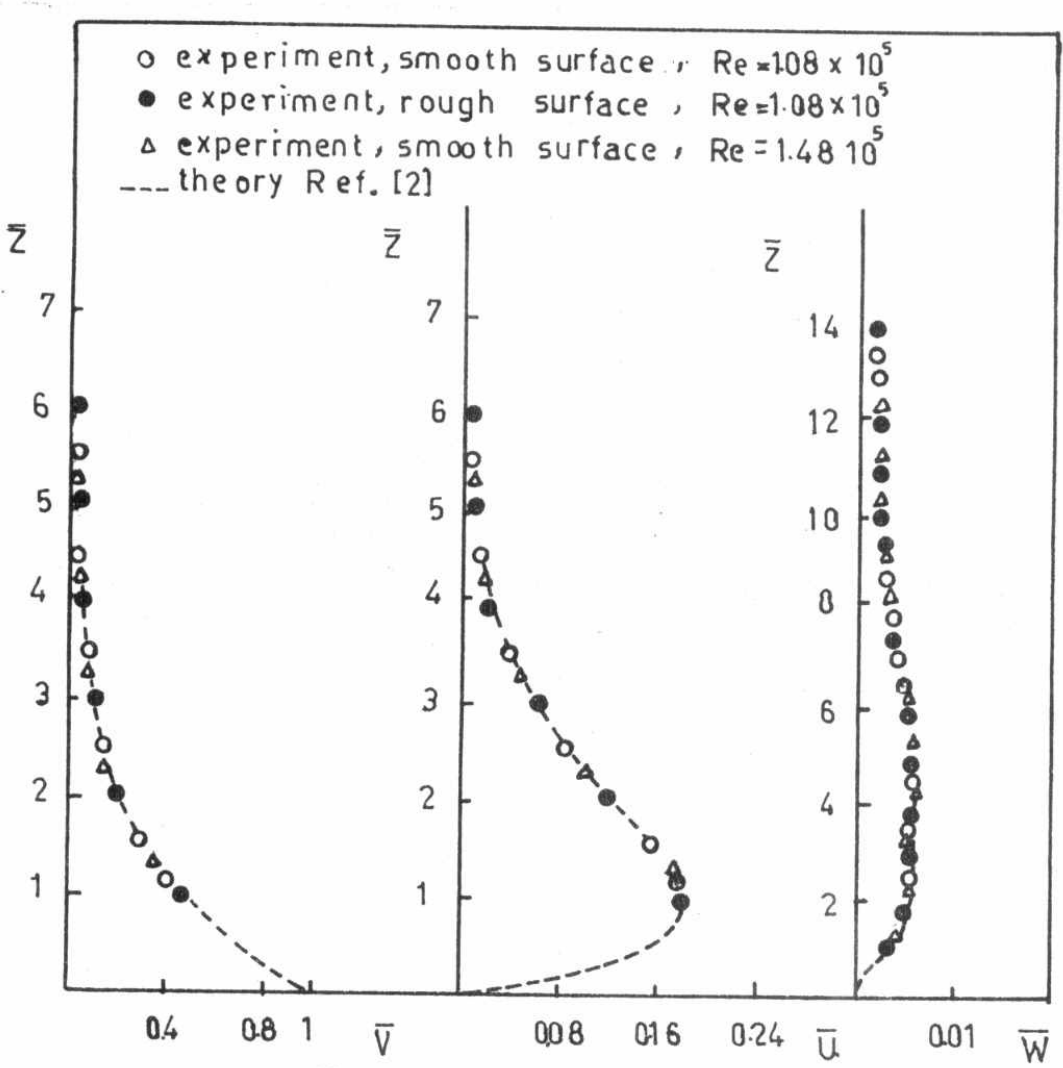

Fig. 4. Flow in the laminar region

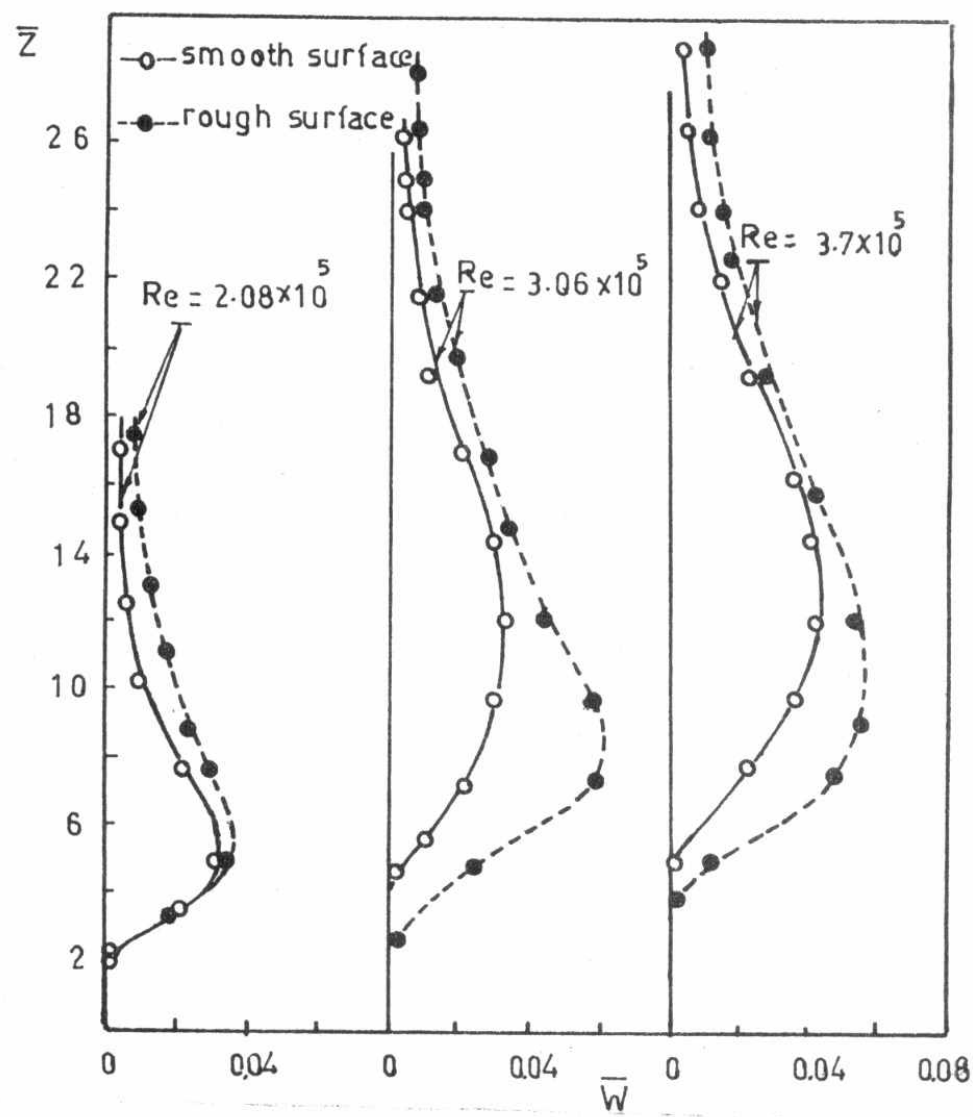

Fig. 5. Axial velocity in the transition and turbulent regions 


\section{\begin{tabular}{l|l}
$E A-7$ & 219
\end{tabular}}

FIRST A.S.A.T. CONFERENCE

14-16 May 1985 , CAIRO
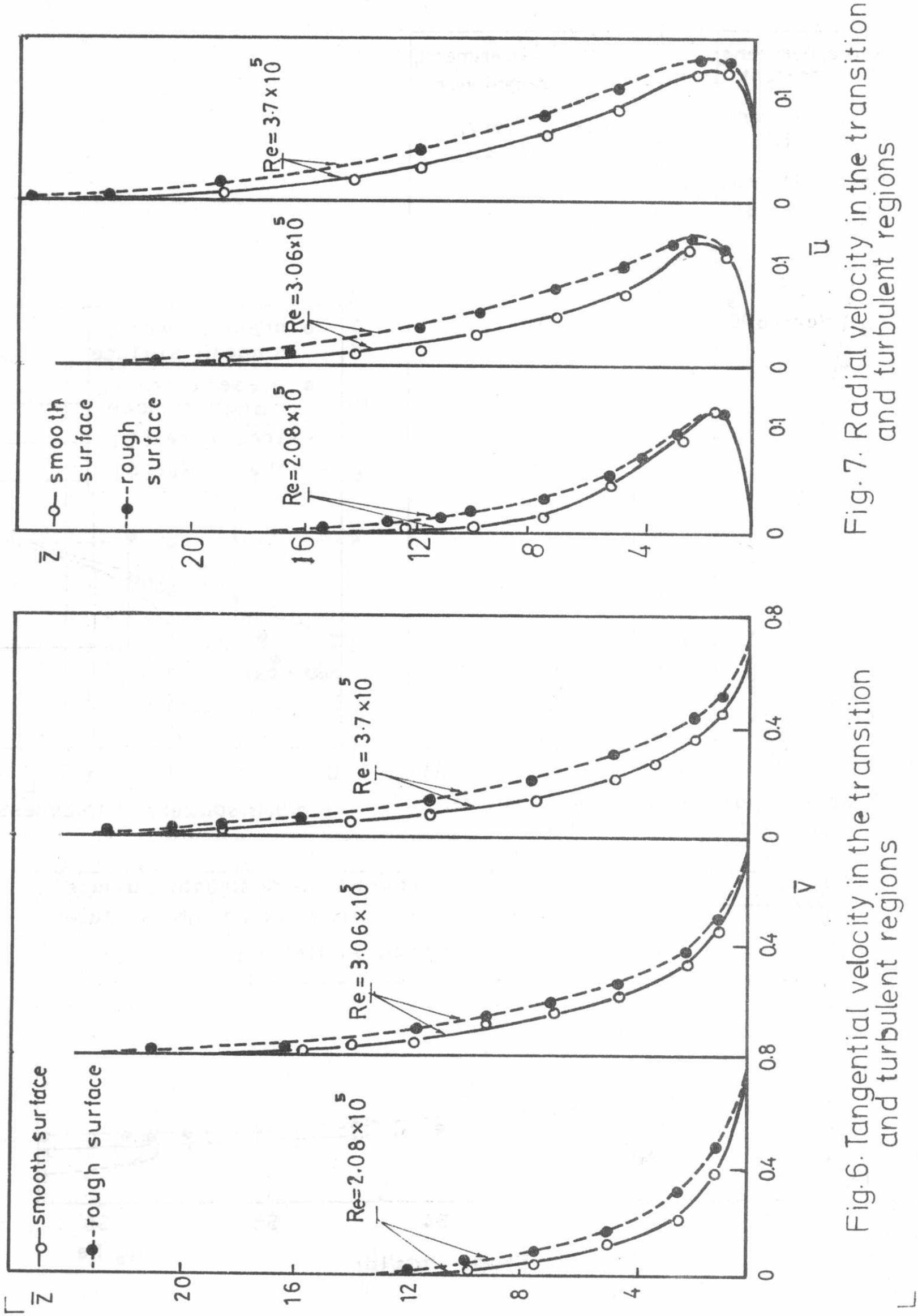


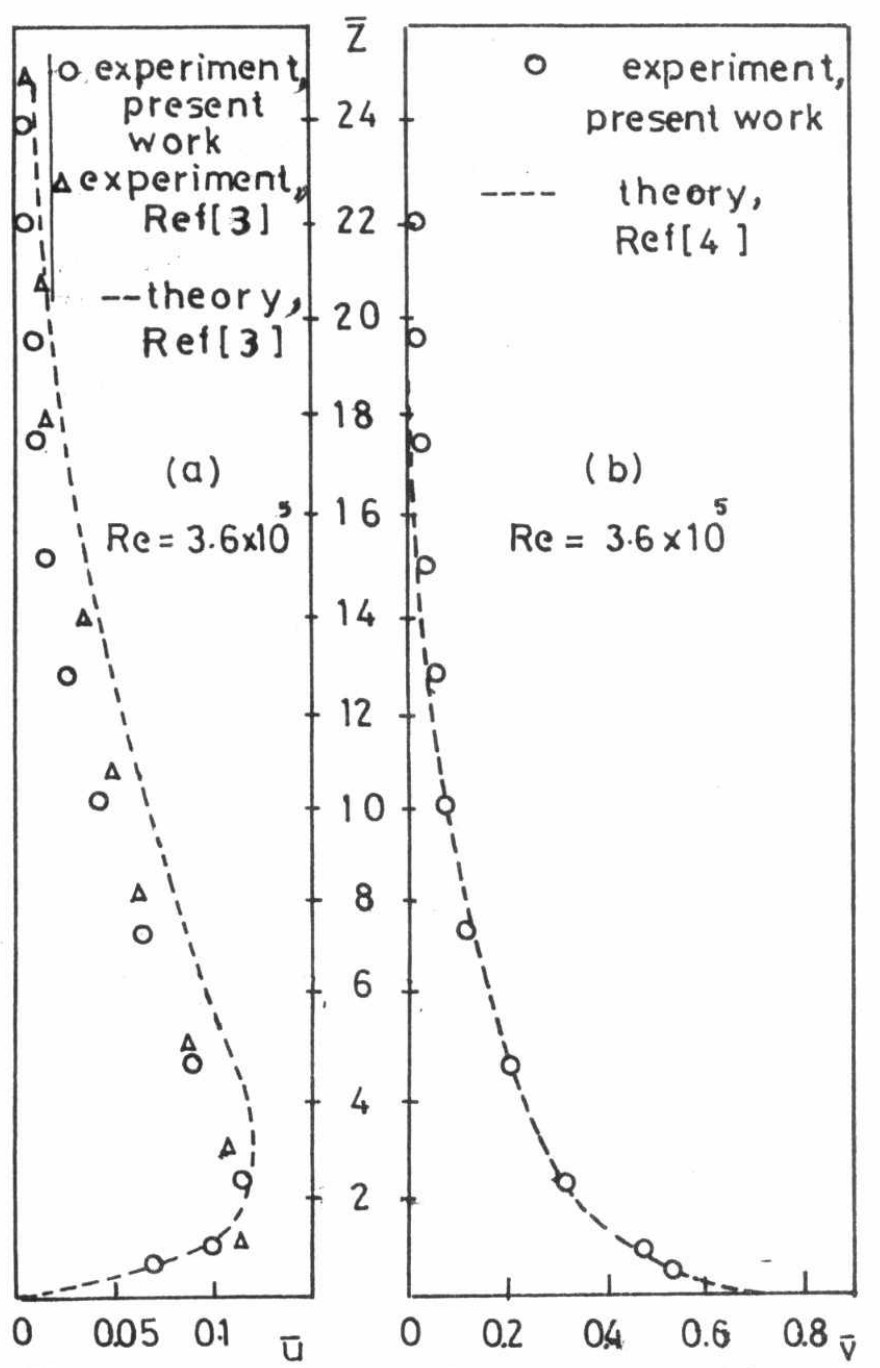

Fig.8. Comparis on of velocity profiles with previous works

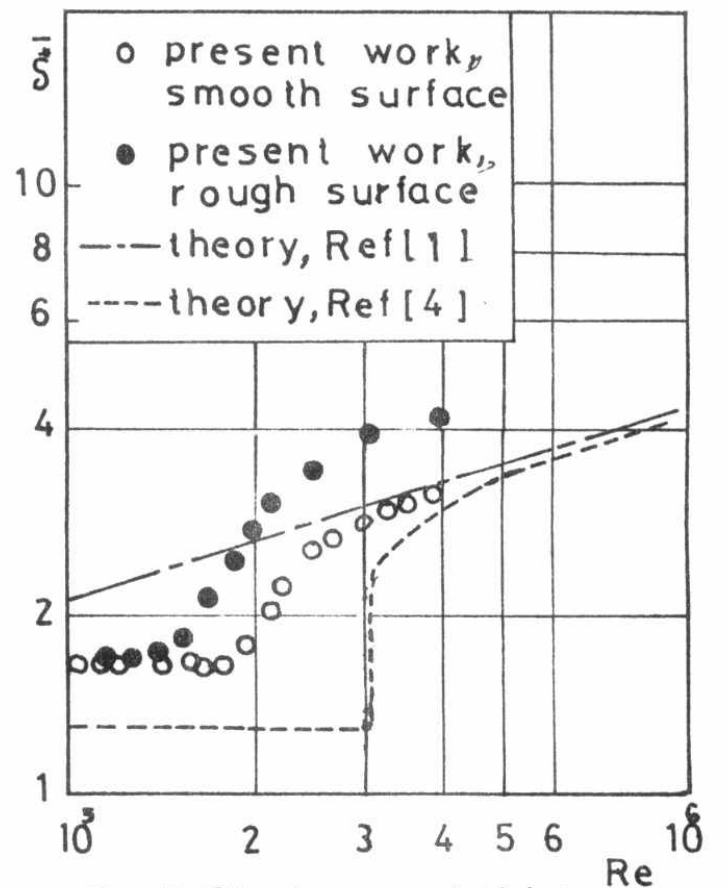

Fig.9. Displacement thickness

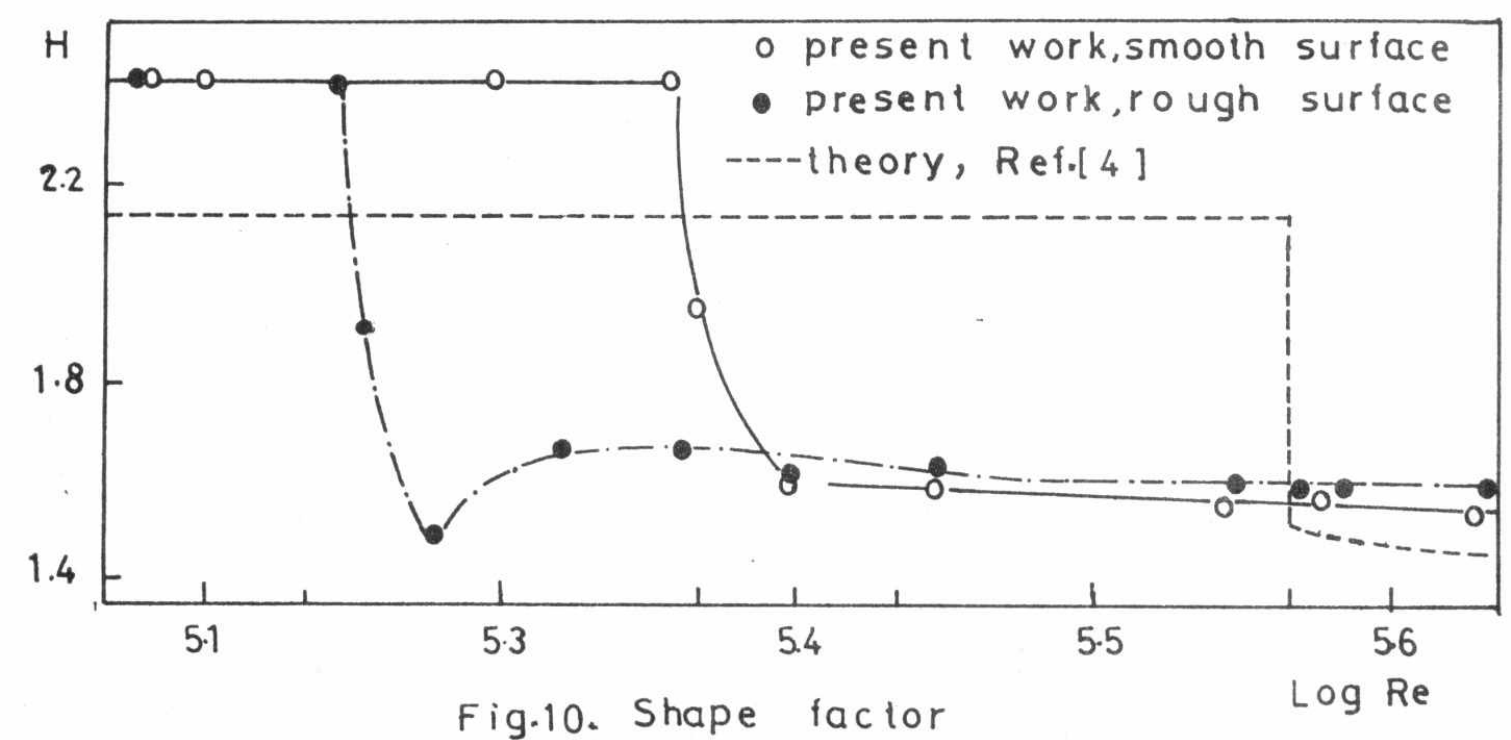

